\title{
Survey of the Performance of Commercial Dose Calibrators for Measurement of ${ }^{123}$ I Activity
}

\author{
Arnold F. Jacobson ${ }^{1}$, Robert Centofanti ${ }^{1}$, Olakiitan I. Babalola ${ }^{1}$, and Brian Dean ${ }^{2}$ \\ ${ }^{1}$ Research and Development, GE Healthcare, Princeton, New Jersey, and Arlington Heights, Illinois; and ${ }^{2}$ Global Supply Chain, \\ Medical Diagnostics, GE Healthcare, Princeton, New Jersey, and Arlington Heights, Illinois
}

The accuracy with which ${ }^{123}$ activity is measured by a dose calibrator depends on the composition and geometry of the source. The present study assessed the variability of current commercial dose calibrators in assaying liquid ${ }^{123}$ | samples. Methods: A calibration procedure for ${ }^{123}$ I measurement was performed on 177 dose calibrators (11 manufacturers) at 138 sites in North America and Europe. Using the standard 123| push-button or dial setting, activity in a 5-mL ${ }^{123}$ | calibration source in a 10-mL U.S. Pharmacopeia type 1 glass vial (actual activity previously determined using a National Institute of Standards and Technology-traceable standard metrology chamber) was measured. A portion of the source was then transferred to a plastic syringe (10-, 5-, and 3-mL sizes at different sites), and the activity in the syringe was measured. Calibration factors (CFs) for converting the dose calibrator readings to the reference activities were then determined for the vial and the syringes. Data were analyzed for all calibrators combined and based on device manufacturers. Measurements using a copper attenuator (sleeve) were made for a subset of 10 dose calibrators at sites that used these devices in clinical practice. Results: Mean CFs for the different measurements were as follows: $10-\mathrm{mL}$ vial, $1.278 ; 10-\mathrm{mL}$ syringe, $0.811 ; 5-\mathrm{mL}$ syringe, $0.815 ; 3-\mathrm{mL}$ syringe, 0.792 . Almost half of the dose calibrators had vial CFs between 1.2 and 1.3 and $10-\mathrm{mL}$ syringe CFs between 0.7 and 0.8 , whereas less than $16 \%$ of the instruments had uncorrected readings within $\pm 10 \%$ of the reference activities. Although there was a wide range of CFs for different calibrators using the copper sleeve, for each unit the CFs for the glass vial and the $10-\mathrm{mL}$ plastic syringe were virtually identical. Conclusion: On most commercial dose calibrators, the standard ${ }^{123}$ s settings result in significant errors in activity measurements for sources in glass vials and plastic syringes. The difference in ionization chamber detection caused by the container composition (glass vs. plastic) is a much larger source of measurement variation than source volume or geometry.

Key Words: dose calibrator; ${ }^{123}$; radioisotope standards

J Nucl Med Technol 2011; 39:302-306

DOI: 10.2967/jnmt.111.089235

Received Feb. 12, 2011; revision accepted May 11, 2011.

For correspondence or reprints contact: Arnold F. Jacobson, GE Healthcare, 101 Carnegie Center, Princeton, NJ 08540.

E-mail: arnold.jacobson@ge.com

Published online Aug. 29, 2011.

COPYRIGHT @ 2011 by the Society of Nuclear Medicine, Inc.
D ose calibrators are used to determine the activity of radiopharmaceuticals administered in nuclear medicine. The displayed activity read-out is based on the ionization current measured as photons emitted by a radioactive source interact with the gas in a sealed, gas-filled ionization chamber. The dose calibrator has no energy discrimination capability; the measured charge reflects the total ionizations produced by interactions of all photons emitted by a particular isotope (1).

It is relatively straightforward to associate ionization current and the activity of a radionuclide with only a single energy photon emission. This process becomes more complex for an isotope with multiple $\gamma$ - and $\mathrm{x}$-ray emissions of different energies. For a dose calibrator to be a practical instrument for use in a clinical setting, it must be capable of automatically converting the measured ionization current into an activity and displaying this result for the user. This is accomplished through the use of isotopespecific calibration factors (CFs) (2). CFs are usually established using standardized sources provided by national standards laboratories. In the United States, the National Institute of Standards and Technology (NIST) supplies these standards.

The primary emission of ${ }^{123} \mathrm{I}$ is a $159-\mathrm{keV} \gamma$-photon, which is emitted with an abundance of approximately $84 \%$ (0.836 photons per disintegration) (3). All other ${ }^{123} \mathrm{I} \gamma$-photons are of higher energy but of low yield (maximum abundance of $1 \%$ ). However, the decay of ${ }^{123} \mathrm{I}$ to ${ }^{123} \mathrm{Te}$ by electron capture is also associated with significant production of K-characteristic x-rays of $27-32 \mathrm{keV}$ (4). These low-energy photons are susceptible to attenuation by the container material, and therefore the measured ionization current in a dose calibrator will be significantly affected by the composition and thickness of the container $(5,6)$. ${ }^{123}$ I capsules routinely used for thyroid studies are sufficiently similar in size and composition to the NIST reference source that the corresponding correction factors stored in the dose calibrator would be expected to be applicable. In contrast, the volume and container composition of ${ }^{123}$ I liquid solutions are considerably more variable. Consequently, dose calibrator correction factors based on the NIST source may not be accurate for liquid ${ }^{123} \mathrm{I}$ measurements. 
The limited clinical use of ${ }^{123}$ I-labeled radiopharmaceutical products in the United States for the past several decades has reduced awareness of the potential need for use of additional correction factors for dose calibrator measurements of ${ }^{123} \mathrm{I}$. However, the recent increase in clinical development and use of injectable ${ }^{123}$ I radiopharmaceuticals has reestablished the need for ensuring accurate activity determinations (7-9). The present study was performed to determine the accuracy of current commercial dose calibrators for measuring ${ }^{123} \mathrm{I}$ activity under conditions routinely encountered in clinical nuclear medicine facilities.

\section{MATERIALS AND METHODS}

A dose-calibrator calibration procedure was performed as part of the initiation process at 138 sites that participated in 3 multicenter international clinical trials of an ${ }^{123}$ I-labeled radiopharmaceutical (10-12). This procedure involved using a 10-mL U.S. Pharmacopeia type 1 glass vial (wall thickness, $2.2[ \pm 0.1] \mathrm{mm}$ ) containing $5 \mathrm{~mL}$ of an ${ }^{123}$ I solution as a reference source. The total activity of each source was determined before shipment to the site. Activity in sources provided to sites in the United States and Canada was measured using a NIST-traceable standard metrology chamber. A comparable instrument was used for activity measurements of sources provided to sites in Europe.

The calibrated activity as of the date and time of measurement was recorded on a certificate included in the shipping container. Because the target activity for the calibration sources provided to sites in the United States

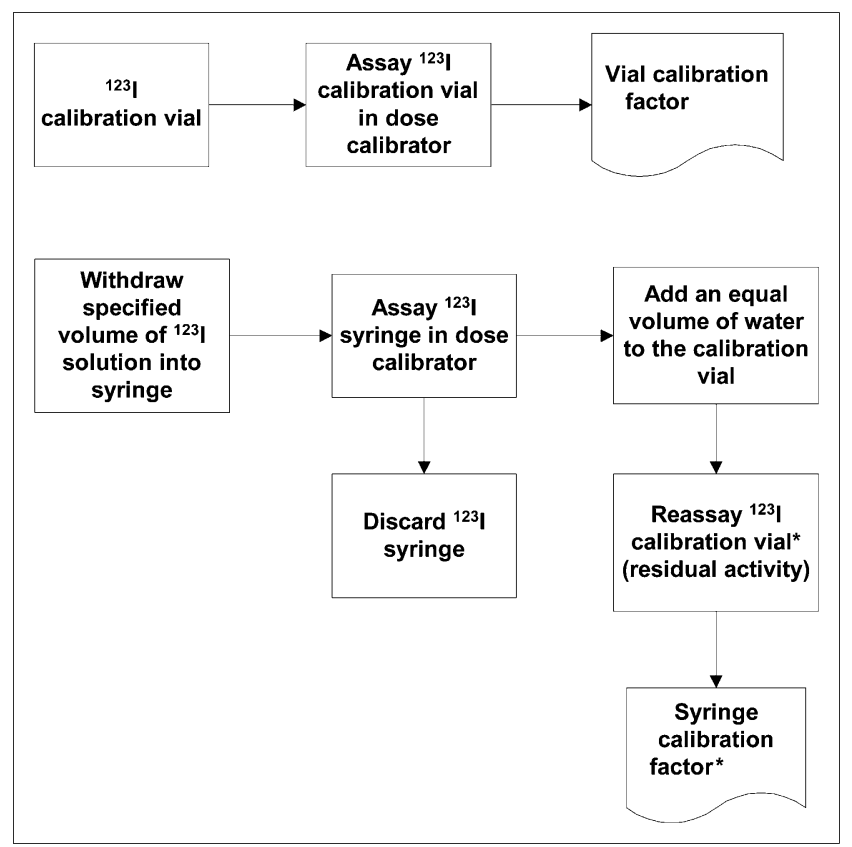

FIGURE 1. Schematic representation of procedures for determination of CFs. Vial CF $=$ (calibrated vial activity $\times$ decay factor)/assayed vial activity. Syringe CF = syringe activity (calibrated vial activity - residual vial activity after syringe withdrawal)/assayed syringe activity. *Syringe and second vial assays were decay-corrected to time of first vial assay.
TABLE 1

Characterization of 177 Dose Calibrators Tested

\begin{tabular}{lcc}
\hline \multicolumn{1}{c}{ Manufacturer } & $\begin{array}{c}\text { No. of } \\
\text { models }\end{array}$ & $\begin{array}{c}\text { No. of } \\
\text { units }\end{array}$ \\
\hline Biodex & 4 & 24 \\
\hline Capintec & 19 & 127 \\
Empos Ltd. & 1 & 1 \\
\hline Medisystem & 1 & 2 \\
\hline MIE Medical Imaging Electronics & 1 & 1 \\
$\quad$ GmbH & 1 & 1 \\
Nuclear Associates & 4 & 9 \\
\hline Nuklear Medizintechnik & 3 & 6 \\
\hline PTW Freiburg GmbH & 1 & 1 \\
\hline Radcal & 1 & 1 \\
\hline Syncor & 3 & 4 \\
\hline Veenstra & & \\
\hline
\end{tabular}

and Canada was $370 \mathrm{MBq}(10 \mathrm{mCi})$ at noon Eastern Standard Time on the following day, the activity at the time of measurement in the metrology chamber was typically $1,480-1,850 \mathrm{MBq}(40-50 \mathrm{mCi})$. In Europe, target activity for sources provided to sites was $37 \mathrm{MBq}$ on the day of dose calibrator measurement.

The procedures performed at each site were designed to assess the performance of the dose calibrator for activity measurements of the original glass vial and solutions in plastic syringes of different volumes. All sites made measurements in a $10-\mathrm{mL}$ syringe, whereas 19 sites that performed nuclear imaging procedures on pediatric patients also tested $5-\mathrm{mL}$ and $3-\mathrm{mL}$ syringes. The details of the measurement procedures are described in the following paragraphs and illustrated schematically in Figure 1.

All measurements were made using the dose calibrator setting for ${ }^{123} \mathrm{I}$ as specified by the instrument manufacturer. This typically involved selecting the appropriate button on the instrument control panel or adjusting the calibration potentiometer to the setting specified in the instrument user manual.

The first measurement was of the glass vial as received from the calibration source provider. The vial was placed in the dose calibrator and the activity reading was recorded, along with the date and time of the measurement, on a worksheet.

The second measurement was made using the $10-\mathrm{mL}$ plastic syringe. A $4.5-\mathrm{mL}$ volume was withdrawn from the vial using a standard long-bore needle (equivalent to that used for drawing up clinical doses), and the needle was then capped. The syringe was placed in the dose calibrator and the activity reading was recorded, along with the date and time of the measurement, on the worksheet. The residual

TABLE 2

CF Summary for 10-mL Glass Vials

\begin{tabular}{|c|c|c|c|c|c|c|}
\hline \multirow[b]{2}{*}{ Container } & \multicolumn{3}{|c|}{ No copper sleeve } & \multicolumn{3}{|c|}{ Copper sleeve } \\
\hline & No. & $\begin{array}{l}\text { Mean } \\
\text { vial CF }\end{array}$ & $\mathrm{SD}$ & No. & $\begin{array}{c}\text { Mean } \\
\text { vial CF }\end{array}$ & $\mathrm{SD}$ \\
\hline 10-mL vial & 167 & $\begin{array}{l}1.278 \text { (range, } \\
0.823-1.757 \text { ) }\end{array}$ & 0.152 & 10 & $\begin{array}{l}1.538 \text { (range, } \\
0.931-2.566 \text { ) }\end{array}$ & 0.697 \\
\hline
\end{tabular}


TABLE 3

CFs for Measurements Using Copper Sleeves

\begin{tabular}{|c|c|c|c|c|}
\hline Dose calibrator & Vial CF & Syringe CF (10 mL) & Difference (syringe CF - vial CF) & Manufacturer and model \\
\hline 1 & 1.288 & Not done & - & Biodex Atomlab 100 \\
\hline 2 & 1.052 & 1.007 & 0.045 & Capintec CRC-15R \\
\hline 3 & 1.663 & 1.628 & 0.035 & Capintec CRC-15R \\
\hline 4 & 2.395 & 2.360 & 0.035 & Capintec CRC-15R \\
\hline 5 & 0.978 & 0.944 & 0.034 & Capintec CRC-15R \\
\hline 6 & 0.976 & 0.971 & 0.005 & $\begin{array}{c}\text { Amersham Radioisotope } \\
\text { Calibrator (ARC)-150 }\end{array}$ \\
\hline 7 & 2.530 & 2.461 & 0.069 & Capintec CRC-15R \\
\hline 8 & 2.566 & 2.492 & 0.074 & Capintec CRC-15R \\
\hline 9 & 0.931 & 0.887 & 0.044 & Capintec CRC-12R \\
\hline 10 & 1.004 & 1.070 & 0.064 & Capintec CRC-15R \\
\hline Mean & 1.538 & 1.536 & 0.002 & - \\
\hline
\end{tabular}

activity in the vial was then determined, with the measurement made after injecting $4.5 \mathrm{~mL}$ of water to reestablish the same volume and geometry as initially. As before, the time of measurement was recorded.

For U.S. sites that performed measurements using 5-mL and 3-mL syringes, the same series of procedures as used for the $10-\mathrm{mL}$ syringe was used. For the $5-\mathrm{mL}$ syringe, the syringe was filled with $2.5 \mathrm{~mL}$, whereas $1.5 \mathrm{~mL}$ was used for the $3-\mathrm{mL}$ syringe. In each case, the vial containing $5 \mathrm{~mL}$ of liquid was measured before and after the syringe measurement in the dose calibrator, with the latter vial measurement performed after water was added to replace the volume drawn into the syringe. For the $5-\mathrm{mL}$ syringe measurement, the original vial activity was about $10 \%$ of that contained in the vial as received by the site (because $90 \%$ had been withdrawn for the $10-\mathrm{mL}$ syringe measurement). Activity in the vial before the $3-\mathrm{mL}$ syringe withdrawal was about $50 \%$ of that before the $5-\mathrm{mL}$ syringe withdrawal.

In Europe, because the calibration vial contained only a low activity, sites that performed measurements on multiple syringes used a separate vial for each determination. The procedures described for the $10-\mathrm{mL}$ syringe were used in each instance, with the lower volumes withdrawn into the smaller syringes as noted.

At some sites, an additional option for ${ }^{123}$ I measurements was the use of a copper sleeve that fits into the well of the dose calibrator. This device attenuates all the low-energy emissions from the isotope, thereby minimizing the effect of container composition $(13,14)$. Sites that used the sleeve clinically performed the same calibration procedures as described above, with all measurements made with the sleeve in place.

The actual activity for each measurement (vial or syringe) was based on the decay-corrected activity of the reference source at the various measurement times. The vial CF was calculated as the decay-corrected vial activity divided by the measured vial activity. The syringe CFs were determined from the difference between the pre- and postfilling vial measurements (using the initially determined vial $\mathrm{CF}$ ) divided by the measured syringe activities (Fig. 1).
All CFs were automatically determined using a computerized worksheet supplied to each site. The CFs for all sites were then entered into a spreadsheet, which included the dose calibrator manufacturer and model.

Descriptive statistics were produced for all dose calibrators and for individual instrument models. Where appropriate, statistical comparisons between groups were performed using Student $t$ tests, with a $p$ value of less than 0.05 considered significant.

\section{RESULTS}

One hundred and 77 dose calibrators from 11 manufacturers at 138 sites were tested. Copper sleeves were used on tests of 10 calibrators at 7 sites. The characteristics of the total sample of dose calibrators are summarized in Table 1.

The CF results for the $10-\mathrm{mL}$ glass vial are summarized in Table 2. Values for vial CF ranged from 0.823 to 1.757 (mean, $1.278 \pm 0.152[\mathrm{SD}]$ ) for measurements made without a copper sleeve and from 0.931 to 2.566 (mean, $1.538 \pm 0.697$ ) with use of a sleeve (difference, $p<0.001$ ). These results indicated that on average, activity in the vial as displayed by the dose calibrator using the standard setting for ${ }^{123} \mathrm{I}$ was significantly underestimated both without

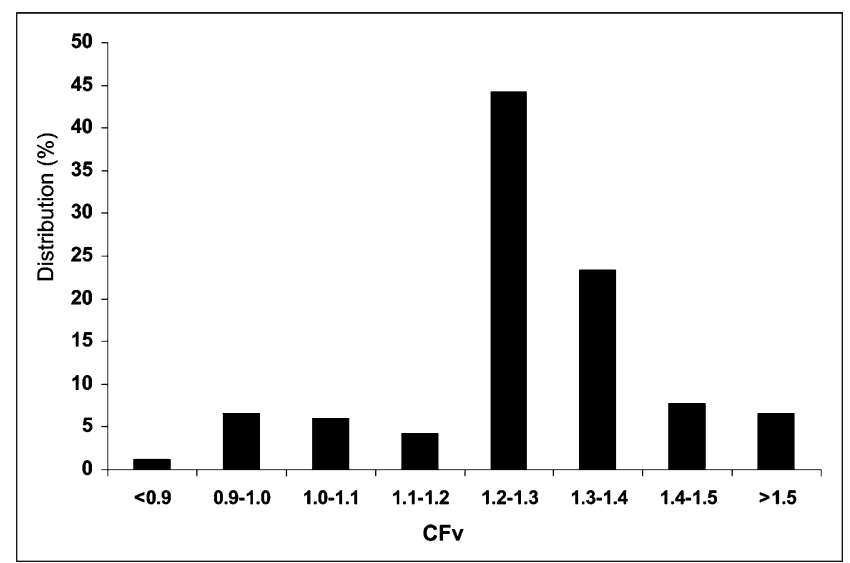

FIGURE 2. Distribution of glass vial CFs for 167 dose calibrators tested without copper sleeves. CFv = vial CF. 
TABLE 4

CF Summary for Plastic Syringes

\begin{tabular}{|c|c|c|c|c|c|c|}
\hline \multirow[b]{2}{*}{ Container } & \multicolumn{3}{|c|}{ No copper sleeve } & \multicolumn{3}{|c|}{ Copper sleeve } \\
\hline & $\overline{\text { No. }}$ & Mean syringe CF & SD & $\overline{\text { No. }}$ & Mean syringe CF & SD \\
\hline 10-mL syringe & 167 & 0.811 (range, $0.472-1.052$ ) & 0.096 & 9 & 1.536 (range, 0.877-2.492) & 0.730 \\
\hline 5-mL syringe & 23 & 0.815 (range, $0.722-1.027$ ) & 0.072 & 2 & 1.030 (range, $1.020-1.040)$ & - \\
\hline 3-mL syringe & 17 & 0.792 (range, $0.728-0.935$ ) & 0.052 & 2 & 0.984 (range, $0.963-1.005$ ) & - \\
\hline
\end{tabular}

and with use of a copper sleeve. Vial CF was greater than 1.20 in $81 \%(136 / 167)$ of dose calibrators tested without copper sleeves and 50\% (5/10) of those tested using sleeves (Table 3). As demonstrated graphically in Figure 2, almost half of the calibrators tested without copper sleeves had a vial $\mathrm{CF}$ between 1.2 and 1.3 and less than $13 \%$ had uncorrected readings within $\pm 10 \%$ of the reference activities.

Syringe CFs for the different volume syringes are summarized in Table 4. The activity in all sizes of syringes was overestimated by a similar amount (mean, $18.9 \%$ for the $10-\mathrm{mL}, 18.5 \%$ for the $5-\mathrm{mL}, 20.8 \%$ for the $3-\mathrm{mL}$ ). For all syringe volumes, the difference between mean vial $\mathrm{CF}$ and syringe CFs was highly significant $(p<0.0001)$. For the copper-sleeve measurements for $10-\mathrm{mL}$ syringes $(n=$ 9), the mean syringe CF was essentially the same as that for vial CF (Table 3). For the $10-\mathrm{mL}$ syringe, almost half the calibrators had a syringe CF between 0.7 and 0.8 , and less than $16 \%$ had uncorrected readings within $\pm 10 \%$ of the reference activities (Fig. 3).

$\mathrm{CF}$ results for the different dose calibrator manufacturers are summarized in Table 5. Although there was some variability between calibrators from different manufacturers, overall variation was similar to that for the most common manufacturer (Capintec) analyzed alone.

\section{DISCUSSION}

Performance characteristics of devices that measure ionizing radiation depend on the manner in which the photon energy is converted into a measurable signal and the accuracy with which that signal is converted into the

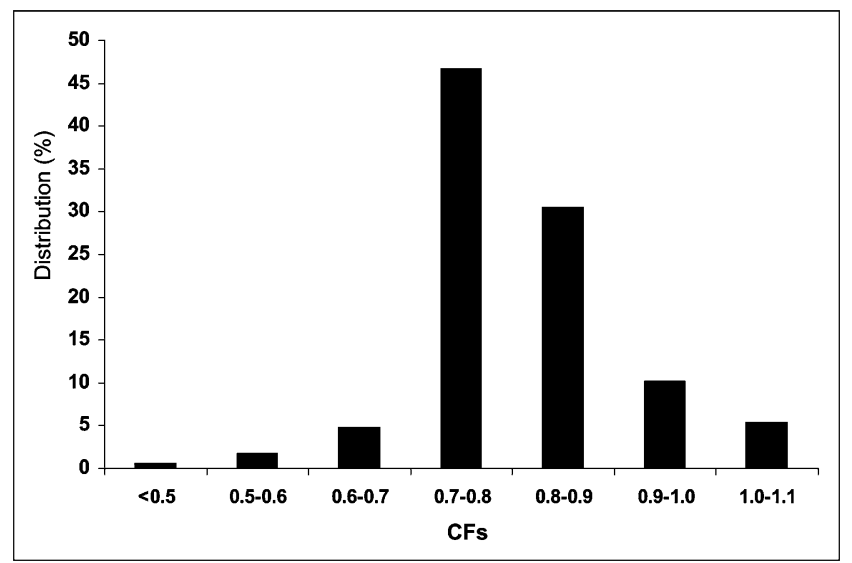

FIGURE 3. Distribution of $10-\mathrm{mL}$ plastic syringe CFs for 167 dose calibrators tested without copper sleeves. CFs = syringe CF. radiologic unit being determined (1). In the case of dose calibrators used to determine radionuclide activity, the conversion of ionization current into disintegrations/s (typically displayed as $\mathrm{mCi}$ or $\mathrm{MBq}$ ) relies on knowledge of the distribution of photons (energy and yield) expected for particular isotopes. The degree to which this distribution can be altered by the geometry of the radioactive source and the composition of its container can affect the accuracy of the measured values provided by the device (4). As demonstrated in the present study, an isotope whose decay results in significant emission of low-energy x-ray and $\gamma$-photons can be particularly challenging for dose calibrator measurements.

For most commercial dose calibrators tested for this report, the difference in readings for a typical activity of ${ }^{123} \mathrm{I}$ used in nuclear imaging (37-370 MBq; 1-10 mCi) when measured in a glass vial versus a plastic syringe was $40 \%-$ $50 \%$, with the result for the former underestimated and the latter overestimated compared with the reference measurement. In the United States, this reflects the use of deviceprogrammed CFs based on the standard NIST ampoule, whose glass thickness $(0.60 \pm 0.04 \mathrm{~mm})$ results in attenuation less than that for the vial but more than that for the syringe. By comparison, for activity measurements of the ${ }^{123}$ I capsules used in thyroid imaging, it is presumed that the standard setting on the dose calibrator provides accurate results, although this was not examined in the present study.

As expected, dose calibrator measurements using a copper sleeve were generally consistent between the glass vial and plastic syringes. Nevertheless, because of the substantial

TABLE 5

Vial CF by Dose Calibrator Manufacturer: No Copper Sleeve

\begin{tabular}{lrc}
\hline \multicolumn{1}{c}{ Manufacturer } & $\begin{array}{c}\text { No. of } \\
\text { units }\end{array}$ & Mean vial CF \\
\hline Biodex & 23 & 1.438 (range, 1.064-1.626) \\
\hline Capintec & 118 & 1.274 (range, 0.823-1.757) \\
\hline Empos Ltd. & 1 & 0.988 \\
\hline Medisystem & 2 & 0.922 (range, 0.890-0.954) \\
\hline MIE Medical Imaging & 1 & 1.312 \\
$\quad$ Electronics GmbH & & \\
\hline Nuclear Associates & 1 & 1.298 \\
\hline Nuklear Medizintechnik & 9 & 1.193 (range, 0.977-1.534) \\
\hline PTW Freiburg GmbH & 6 & 1.069 (range, 0.917-1.207) \\
\hline Radcal & 1 & 1.227 \\
\hline Syncor & 1 & 1.224 \\
Veenstra & 4 & 1.210 (range, 0.996-1.332) \\
\hline
\end{tabular}


attenuation of $159-\mathrm{keV}$ photons by this device, it is still necessary to apply a correction factor to the activity reading to obtain accurate values. It is likely that the 5 dose calibrators with CF results near 1.0 with use of the copper attenuator had been previously adjusted to incorporate such a correction factor. However, given the small number of centers that had and used these sleeves, the scope of the current report with regard to assessment of consistency across the spectrum of dose calibrators is limited.

Most dose calibrators provided consistent readings for the different syringe sizes and volumes, indicating that the effect of source geometry was small relative to that of the container composition. Thus, most users of liquid ${ }^{123} \mathrm{I}$ radiopharmaceuticals need determine the $\mathrm{CF}$ for only 1 syringe size to obtain accurate measurements of radionuclide activity.

This study involved one of the largest comparative evaluations of dose calibrators of which we are aware $(6,15)$. The degree of discordance between measured and actual ${ }^{123}$ I activity in the present study is similar to that reported in several surveys of dose calibrators in the United Kingdom $(6,15)$. Nevertheless, because most of the instruments tested in the current evaluation were manufactured by 2 companies, the results cannot be specifically applied to all the less common dose calibrators that are in clinical use. This factor is somewhat less of a concern because of the design similarities among all dose calibrators and the fact that the principles governing conversion of $\mathrm{x}$ - and $\gamma$-ray photon energy to ionization current in a gas-filled chamber are universal. The devices sampled and the data collected in this study provide a reasonable representation of the dose calibrators in current clinical use.

Although the results of the present study have implications for clinical use of dose calibrators for measurement of ${ }^{123}$ I-labeled radiopharmaceuticals, they do not indicate a need for changes in routine quality control testing of dose calibrators. Daily constancy, quarterly linearity, and annual accuracy tests should detect device drift that could affect the accuracy of the ${ }^{123}$ I CF. If the accuracy test shows a larger than expected difference between measurements of low- and high-energy isotope sources, it would be prudent to perform a new determination of the ${ }^{123}$ I CF. Similarly, the CF should be determined subsequent to repair of a dose calibrator or installation of a new instrument.

\section{CONCLUSION}

There is a wide variation in measurement results when assaying liquid ${ }^{123} \mathrm{I}$ in commercial dose calibrators, with the container composition (glass vs. plastic) being a much larger source of measurement variation than source volume or geometry. As such, dose calibrator-specific CFs are needed to accurately determine the ${ }^{123} \mathrm{I}$ activity administered to the patient. A copper sleeve can eliminate the CF difference between different source containers, but properly determined CFs are still necessary. As an adjunct to routine quality control testing of dose calibrators, the ${ }^{123} \mathrm{I}$ CF should be determined before the initial use of injectable ${ }^{123}$ I-labeled radiopharmaceuticals and after any instrument repairs.

\section{ACKNOWLEDGMENT}

This study was performed by GE Healthcare. All authors were employed by GE Healthcare at the time the data for the study were collected or analyzed. No other potential conflict of interest relevant to this article was reported.

\section{REFERENCES}

1. Sorenson JA, Phelps ME. Physics in Nuclear Medicine. 2nd ed. Orlando, Florida: Grune \& Stratton, Inc; 1987:294-297.

2. Capintec Model CRC-15R Radioisotope Dose Calibrator Owner's Manual. Ramsey, NJ: Capintec Inc; 1990.

3. Dillman LT, Von der Lage FC. Radionuclide Decay Schemes and Nuclear Parameters for Use in Radiation-Dose Estimation: NM/MIRD Pamphlet 10. Reston, VA: Society of Nuclear Medicine; 1975:68.

4. Harris CC, Jaszczak RJ, Greer KL, Briner WH, Coleman RE. Effects of characteristic X-rays on assays of I-123 by dose calibrator. J Nucl Med. 1984;25:13671370 .

5. Harris CC, Jaszczak RJ, Greer KL, Briner WH, Coleman RE. Solution to problems in dose calibrator assays of iodine-123. Am J Physiol Imaging. 1988;3:33-35.

6. Tyler DK, Woods MJ. Syringe calibration factors for the NPL secondary standard radionuclide calibrator for selected medical radionuclides. Appl Radiat Isot. 2003;59:367-372.

7. Tamaki N, Yoshinaga K. Novel iodinated tracers, MIBG and BMIPP, for nuclear cardiology. J Nucl Cardiol. 2011;18:135-143.

8. Bombardieri E, Coliva A, Maccauro M, et al. Imaging of neuroendocrine tumours with gamma-emitting radiopharmaceuticals. Q J Nucl Med Mol Imaging. 2010;54:3-15.

9. Bairactaris C, Demakopoulos N, Tripsianis G, et al. Impact of dopamine transporter single photon emission computed tomography imaging using I-123 ioflupane on diagnoses of patients with parkinsonian syndromes. J Clin Neurosci. 2009; 16:246-252.

10. Vik TA, Pfluger T, Kadota R, et al. ${ }^{123} \mathrm{I}-\mathrm{mIBG}$ scintigraphy in patients with known or suspected neuroblastoma: results from a prospective multicenter trial. Pediatr Blood Cancer. 2009;52:784-790.

11. Wiseman GA, Pacak K, O'Dorisio MS, et al. Usefulness of ${ }^{123}$ I- $m$ IBG scintigraphy in evaluation of patients with known or suspected primary or metastatic pheochromocytoma or paraganglioma: results from a prospective multi-center trial. J Nucl Med. 2009;50:1448-1454.

12. Jacobson AF, Lombard J, Banerjee G, Camici P. ${ }^{123} \mathrm{I}-m \mathrm{IBG}$ scintigraphy to predict risk for adverse cardiac outcomes in heart failure patients: design of two prospective multicenter international trials. J Nucl Cardiol. 2009;16:113-121.

13. Wiarda KS. Use of a copper filter for dose calibrator measurements of nuclides emitting K x-rays. J Nucl Med. 1984;25:633-634.

14. Kowalsky RJ, Johnston RE. Dose calibrator assay of iodine-123 and indium-111 with a copper filter. J Nucl Med Technol. 1998;26:94-98.

15. Tyler DK, Baker M, Woods MJ. NPL secondary standard radionuclide calibrator: syringe calibration factors for radionuclides used in nuclear medicine. Appl Radiat Isot. 2002;56:343-347. 